\title{
Biomimetic Middleware Design Principles for IoT Infrastructures
}

\section{Zenon Chaczko ${ }^{1}$, Ryszard Klempous ${ }^{2}$, Jerzy Rozenblit ${ }^{3}$, Tosiron Adegbija ${ }^{3}$, Christopher Chiu ${ }^{1,2^{*}}$, Konrad Kluwak ${ }^{2}$, Czeslaw Smutnicki ${ }^{2}$}

${ }^{1}$ University of Technology, Sydney, 15 Broadway, Ultimo, NSW 2007, Australia zenon.chaczko@uts.edu.au,christopher.chiu@uts.edu.au

${ }^{2}$ Faculty of Electronics, Wrocław University of Science and Technology, Wybrzeże Wyspiańskiego 27 50-370 Wrocław, Poland ryszard.klempous@pwr.edu.pl,konrad.kluwak@pwr.edu.pl, czeslaw.smutnicki@pwr.edu.pl

${ }^{3}$ The University of Arizona, Dept. of Electrical and Computer Engineering, 1230 East Speedway Blvd. P.O. Box 210104, Tucson, AZ 85721-0104, USA jr@ece.arizona.edu, tosiron@email.arizona.edu

Abstract: The advancement of Internet of Things (IoT) has made it practical to discover, localize and pinpoint smart sensing devices based on the situational context, relevancy, and characteristics to query data intelligently, or conduct actions. Furthermore, the development of large-scale applications must deal with data collection and data sensing from a massive number of ubiquitous components, ultimately converging into $5 G$ mobile networking. Additionally, IoT involves managing the expectations of Big Data sourced from many heterogeneous sources. This paper provides an overview of biomimetic methodologies, which represent a viable solution for large-scale data delivery through the aggregation of information with large-scale IoT technologies.

Keywords: $5 \mathrm{G}$ communications; big data; Internet of Things; machine-to-machine systems; massive-scale systems; middleware; ubiquitous systems; wireless sensor and actuator networks

\section{Introduction to IoT-based Middleware}

The Internet of Things (IoT) presents several potential research opportunities and raises development challenges in modern computing [7]. Technological advancements stimulate a new paradigm where sensors and actuators are managed

1,2* On leave from University of Technology, Sydney 
as common infrastructure, offering multiple concurrent services and intelligent data aggregation to many users [6], [12].

Advancements driving the massive-scale IoT infrastructure concept include:

- Autonomic Computing [1] and Middleware Design [3];

- Smart Wireless Sensor and Actuator Networks (WSANs);

- Artificial Intelligence (AI);

- Communication Protocols and Standards; and

- Fifth Generation Mobile Cellular Networks (5G).

Along with the deployment of $5 \mathrm{G}$ networking, the opportunity presented in the realm of IoT and multimedia sphere includes responsive connectivity for Machine-to-Machine systems (M2M), and possibilities into near real-time interactive services. It is also important to consider practical $5 \mathrm{G}$ rollout challenges when adopting multimedia interactivity such as latency and bandwidth constraints.

In addition, the significance that IoT places on $5 \mathrm{G}$ regarding future demand must be considered, using the experiences of current cellular utilization. Rapidly advancing services including Augmented Reality (AR), Virtual Reality (AR) and multi-user conferencing must be factored into the IoT middleware as part of the architectural framework.

The approach is different when compared to dedicated systems forming part of legacy telecommunications and computer infrastructures. Much research needs to be considered to adapt standard protocols, middleware model standards, data aggregation. and methods of sensor fusion as well as mechanisms of data delivery, to configure the approach IoT can integrally build into public infrastructures. The emergence of new communication technologies requires adaptable solutions that handle M2M connectivity while minimizing harmful downtime interruptions.

The solution is an architectural model for implementing the software-directed paradigm known as the Biomimetic Model of Middleware (BMM) [5]. The localization and topology of middleware components in the IoT software solution is shown in Figure 1. The components are IoT devices, local and, core middleware and Internet connectivity. 


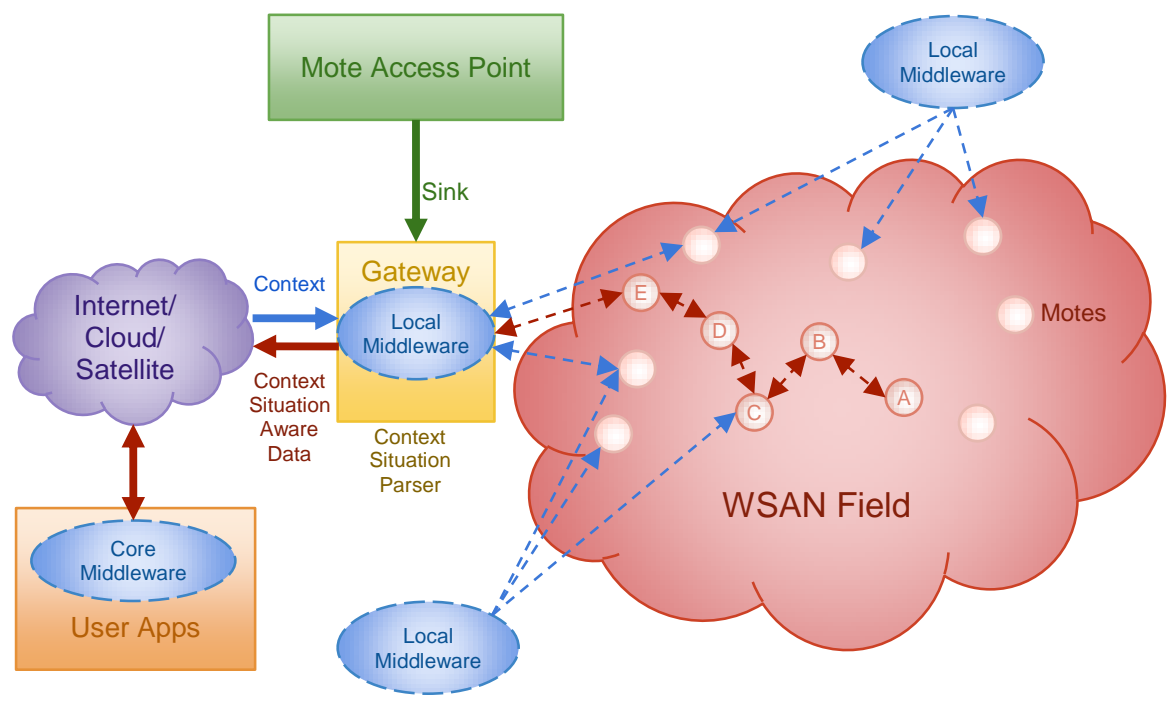

Figure 1

IoT Middleware Architecture featuring Biomimetic Principles

For IoT nodes, Mote Access Point (MAP) devices have two main responsibilities:

1. Manage IoT devices while also partitioning the core functions of different components and ensuring that every component runs effectively:

a. Sensors as data sinks, and

b. Actuators as control sinks;

2. Act as intelligent access points with router functionality at the sensor/control boundary of IoT devices, which are composed of context aware:

a. Data parsers, and

b. Gateway processors.

The research work reflects the innovative form of biomimetic middleware for IoT infrastructures, along with the complementary models for data aggregation. The biomimetic middleware enables the generation of adaptable WSANs and services that lead to practical viability [5].

By providing a discussion on the design concerns of IoT-based middleware, the potential innovations include the broader vision of large scale, advanced softwareintensive IoT infrastructures for modern computation and connectivity facilitating smart M2M communications.

In this work, we propose a Biomimetic Model of Middleware (BMM), along with adaptive techniques for data aggregation. Next, we explore biomimetic 
middleware design aspects that enable the development and deployment of contextually aware, heterogeneous, secure, and massive-scale system solutions [8].

Finally, we provide a practical illustration to show the viability and feasibility of implementing BMM for IoT infrastructures, showing the possibilities to deploy the BMM approach from a broad-based perspective. This is important, given the role of software in the field of Software Defined Networking (SDN) solutions that plays a critical role in $5 \mathrm{G}$ cellular communications.

\section{Biomimetic Middleware Models}

Examples of technological advancements that can enable biomimetic solutions in various technical fields are shown in Table 1 [2]. Progress in modern technologies is driven by biomimetic forms in design in the areas of advanced manufacturing, cloud computing infrastructures and open-learning AI frameworks. These sectors will play a critical role in the design, implementation, and deployment of IoT infrastructures.

Table 1

Biomimetic Solutions in Modern Industrial Implementations

\begin{tabular}{|c|c|c|}
\hline Field & Implementation & Biomimetic Solution \\
\hline \multirow{2}{*}{$\begin{array}{l}\text { Advanced } \\
\text { Manufacturing }\end{array}$} & Additive Manufacturing & 3D, 4D, 5D Printing \\
\hline & Kirigami Construction & Additive Lattice Structures \\
\hline \multirow{2}{*}{$\begin{array}{l}\text { Cloud } \\
\text { Computing }\end{array}$} & AI and Machine Learning & AIaaS, MLaaS Services \\
\hline & Containers, Funtainers & CaaS, FaaS Services \\
\hline \multirow{6}{*}{$\begin{array}{l}\text { Learning } \\
\text { Frameworks }\end{array}$} & AI \& Deep Learning & Intel AI Framework, BigDL \\
\hline & Flow Graph Computation & CNTK, TensorFlow \\
\hline & Image Classifier Processing & Caffe \& Caffe2 \\
\hline & Natural Language Libraries & Gensim \\
\hline & $\begin{array}{l}\text { Neural Network } \\
\text { Frameworks and Dynamic } \\
\text { Parallelization }\end{array}$ & $\begin{array}{l}\text { Apache Mxnet, Chainer, } \\
\text { CMU Dynet, Keras, Paddle, } \\
\text { Pytorch \& Torch }\end{array}$ \\
\hline & Statistical Data Processing & Gluon, H2O, RStudio \\
\hline
\end{tabular}

\subsection{Advanced Manufacturing}

\subsubsection{Additive Manufacturing}

Additive manufacturing has guided the design and construction of uniquely complex structures that would be impossible with traditional subtractive manufacturing. Technologies such as 5D printing can readily build sophisticated, 
multi-faceted designs that will propel innovative technical approaches within a short lead time.

By enabling the rise of rapid prototyping in IoT infrastructures, additive manufacturing allows designers and engineers to build IoT solutions while minimizing construction and testing outlay. Thus, BMM infrastructure deployment is accomplished as IoT mote and gateway designs can be easily revised for a specific context while maintaining cost efficiency.

\subsubsection{Kirigami Structures}

Kirigami is the Japanese art of folding paper objects that can be unfurled into elaborate structures. The strategic fold points maximize the compressibility of the structure when compacted, but once unfolded reveals its true form. Thus, the unfolded form serves the genuine intention of the structure, operating its true functionality.

This approach is applicable for IoT, where the design and deployment can take place in confined environments, or in restricted domains where total space is at a premium. Thus, it is compressible into a stored manner when not used, or expandable as necessary such as maximizing signal strength during radio congestion.

\subsection{Cloud Computing}

\subsubsection{Artificial Intelligence and Machine Learning Services}

The adoption of AI cloud platforms by academia and industry is driven by the low-cost of entry and simplicity of provisioning resources. Corporate providers including Amazon Web Services, Google Cloud and Microsoft Azure offer ondemand turnkey services that include open source libraries with broad accessibility and technical support.

As AI and ML cloud services migrate to Systems on a Chip (SOC) and Application Specific Integrated Chipset (ASIC) solutions, this has led to highly optimized computing efficiency gains that lower the total cost of ownership for IoT-based platforms that cannot feasibly be achieved using general purpose computing infrastructure.

The net result of these industry trends means tailored big data solutions are deployable remotely from the IoT cloud, to reach optimum economies of scale for computing resources, and thus satisfy peak operational storage and processing efficiency. 


\subsubsection{Containers and Funtainers}

In conjunction with container-based solutions in the cloud, these solutions provide a highly encapsulated environment where all functional libraries are included in the virtual computing space. Therefore, one can rapidly deploy functionally similar components experimentally that is less prone to configuration errors and other constraints.

By enabling rapid software prototyping, it is advantageous for designing IoT infrastructures where an adaptable BMM can be immediately deployed. The testing and results of the solution can be assessed and collated for further investigation, with the development of new $\mathrm{AI}$ and $\mathrm{ML}$ algorithms to evaluate using IoT infrastructure.

\subsection{Learning Frameworks}

Collaborations between industry and academic research sectors have resulted in the development of open-source frameworks for machine learning. These collaborations have enabled a wide variety of datasets and algorithms suitable for IoT middleware solutions, along with their combined experience of development skills and expertise. Examples of open-source frameworks contributed by the academic and industrial communities include Apache Mxnet, Caffe, Caffe2, Chainer, CMU DyNet, DSSTNE, Gensim, Gluon, H2O, Intel AI Framework, Intel BigDL, Keras, Microsoft CNTK, Paddle, Pytorch and Torch, RStudio and TensorFlow.

Machine Learning (ML) has been applied to various fields including medicine, precision agriculture, sports science, and entertainment. Thus, ML is applicable for IoT deployment where node routing and management must be achieved efficiently while preserving the energy constraints of mote batteries. Hence, IoT middleware design can be implemented to accomplish tailored tasks including recognition of patterns for industrial and government applications, or in surveillance, where security patrol can be augmented with AI recognizers that aid in repetitive operations such as feature identification.

Recognition of patterns within Computer Vision (CV) enhancement is feasible, especially where compute capability is limited due to energy constraints. This is because the trend of mobile multi-core processors has reached maturity, such that elementary AI tasks can be performed at the edge gateway. The development of a hierarchical middleware model breaks down computational tasks in an intelligent manner where low-demand operations are performed on-field, while computationally complex big data tasks are done in the cloud, as illustrated in Table 2 [10]. 
Table 2

AI Operations for an IoT-based Context

\begin{tabular}{|l|l|l|}
\hline Operational Location & Compute Device & AI/ML Examples \\
\hline On-field/site: & IoT Devices: & Unsupervised Learning: \\
Local Operations & $\bullet \quad$ WSAN End Points & $\bullet \quad$ Self-Organized Maps \\
& $\bullet$ Edge Gateway & $\bullet$ Neural Networks \\
\hline Off-field/site: & Cloud Infrastructures: & Supervised Learning: \\
Remote Operations & $\bullet$ AIaaS & $\bullet$ Genetic Algorithms \\
& $\bullet$ MLaaS & $\bullet$ Generative \\
& & Adversarial \\
& & Networks \\
\hline
\end{tabular}

\subsubsection{On-field Operations}

On-field AI tasks complementing an IoT context include unsupervised learning with Self-Organized Maps (SOM) and neural networks. Such computational tasks would not significantly burden the energy, data, and computational constraints of the IoT gateway. The middleware needs to enable priority to unsupervised learning operations for the localized IoT infrastructure, since the effort for greater computation tasks should be done remotely where resources are more plentiful, thus adding to system redundancy [14].

\subsubsection{Off-field Operations}

Off-field AI tasks accompanying an IoT context include supervised learning using complex algorithms, such as Genetic Algorithms (GAs). AIaaS (AI-as-a-service) and MLaaS (machine learning-as-a-service) infrastructures are suitable since they are tasked with heavy-duty compute and resource needs [15], [16]. Tailoring AIaaS and MLaaS solutions include denormalized databases suited for long-term archiving and monitoring of IoT data. This is essential for big data tasks such as long-term trending analysis and predictive forecasting capabilities relying on large datasets for statistical probabilities.

\section{Applications of Biomimetic Middleware Solutions}

\subsection{Integration of IoT with Motion Capture}

The application of biomimetic design in middleware can be observed from IoT solutions in the field of human motion tracking. This field of research has evolved to the point where sensing technologies are commercially embedded such as Apple FaceID, Microsoft Kinect and Sony SoftKinetic. These platforms facilitate AR and VR applications for domestic and industrial applications. 
The importance of consumer adoption should not be underestimated, as adoption drives economies of scale and mainstream adoption. Thus, combining commercial motion capture technologies with IoT provides the potential for rapid deployment, while also reducing the total cost of ownership concerns. The momentum of adoption leads to acceptance and familiarity as part of broader IoT middleware design.

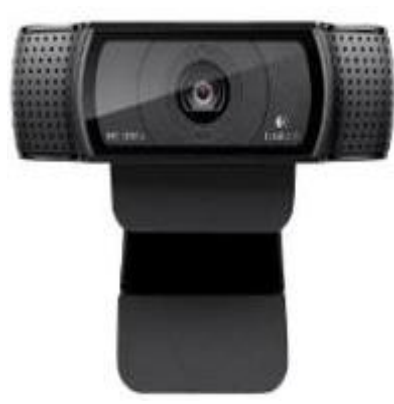

High-Definition Web Camera

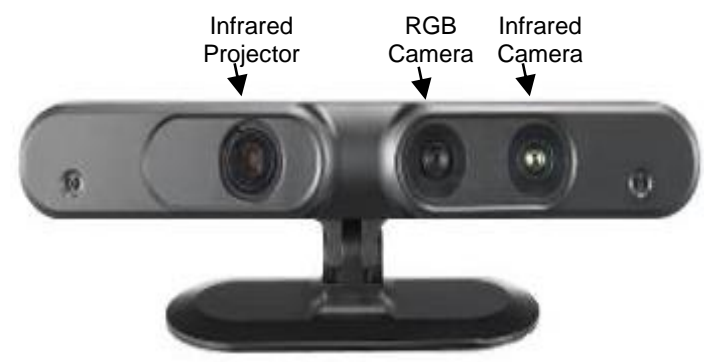

Infrared Time-of-Flight Camera

Figure 2

Current motion capture systems for consumer applications

Examples of motion capture systems that are common in consumer gaming and entertainment are shown in Figure 2. They include arcade systems, set-top boxes, and interactive multimedia where an immersive visual communication medium can complement IoT-based solutions for environmental monitoring applications.

In addition, the professional arena has also been complemented with motion capture technologies where sophistication and high precision levels are required. Vendor solutions from North America and the European Union include Contemplas, GaitTrack, Qualisys and TekScan. These tools have a high outlay along with recurring maintenance expenses that constrain broader adoption.

Hence consumer-based motion capture solutions are best matched for IoT middleware application, because greater adoption of IoT with M2M communications will take place as $5 \mathrm{G}$ rollouts become commonplace. Minimizing maintenance concerns such as cost of replacement will increase the adoption rate and end user accessibility.

\subsection{End User Factor within IoT Middleware}

The IoT middleware platform must be developed to account for quality control, especially in the field of motion capture and annotation analysis to ensure system implementation flexibility.

Connecting the consumer arena when factoring the number of motion tracking systems available in the marketplace, as well as the proprietary nature of 
professional motion capture systems, means that a complementary and practical application must reasonably adopt mainstream design principles.

Thus, the complete IoT-based solution integrating biomimetic middleware with motion capture sensors must aim to be technology and vendor agnostic. The solution should feasibly adopt open source software and technologies for broader developer adoption and ease integration with newly evolving IoT development platforms.

Therefore, the middleware IoT infrastructure is not simply glue logic to combine components into a common space, but handle massive-scale IoT heterogeneous concerns in an ubiquitous manner. Wider reach of an IoT-based BMM solution is achieved when it provides a M2M communications mechanism, as well as professional and consumer interactivity in a seamless manner.

\subsection{Innovations in IoT Middleware Research}

The conception of the BMM technology platform in the scope of motion capture should consider cooperation with academic and institutional organizations for scientific research and innovation. This is achieved by building the IoT middleware framework with a customizable architecture that is not linked to an industry or commercial design goal.

The place where application-specific analysis is done at the AIaaS and MLaaS backend, such that separation is ensured between computational processors and IoT nodes. Since there are many motion capture applications that can be applied with IoT, including television broadcasts, motion pictures, sports physiotherapy, and tele-medicine, the functional separation of processing concerns is noteworthy in this research.

In this work, as a case study, we explore the application of biomimetic principles to middleware for analysis of healthcare employees. The practical piecemealing of operations in the healthcare domain means the middleware infrastructure is segmented into component-level work tasks for logical deployment.

Hence, the case study will examine the daily requirements of healthcare employees. By combining biomimetic principles using IoT, there is the potential to accommodate workplace training needs in alliance with occupational therapists including physiotherapists. 


\subsection{Case Study Adopting BMM IoT Middleware}

\subsubsection{Healthcare Employee Training and Analysis}

\subsubsection{Lifting Practice and Analysis Problem Space}
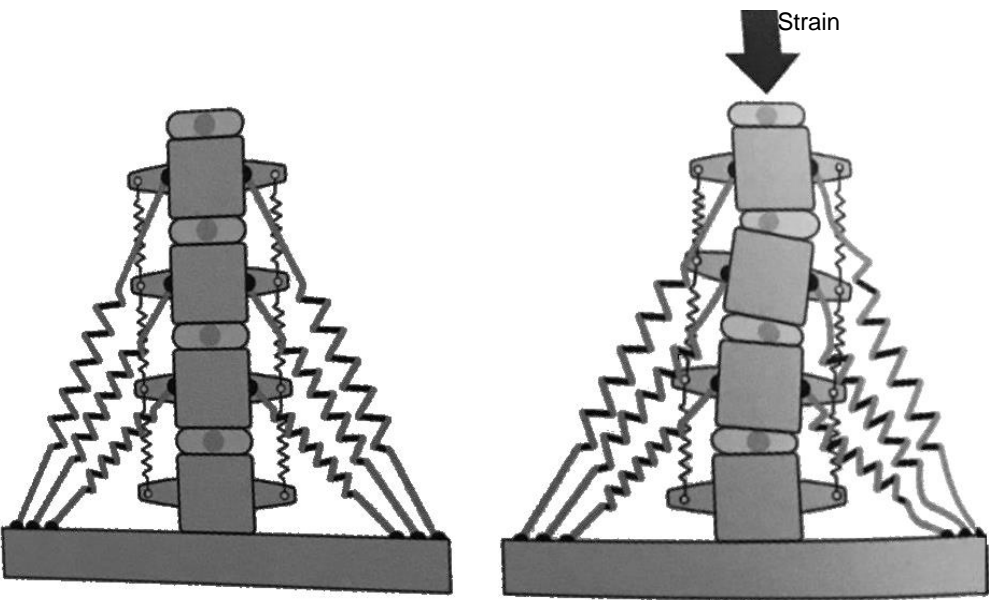

Figure 3

Depiction of back strain resulting from incorrect lifting technique [17]

Investigative research conducted by the Wrocław Medical University identified that employees would benefit from a posture analysis system to identify incorrect lifting practice. The worldwide increase in back injuries resulting from lifting objects greater than $25 \mathrm{~kg}$ has led to costs associated with employee downtime including compensation and rehabilitation [9].

The emotional and financial burden is anticipated to rise as population aging occurs in modern society, placing further stress on existing employee workloads. The best way to manage this emerging issue is through improving training and rehabilitation protocols, thus enforcing positive lifting practices in medical facilities.

Thus, as patient care duties inevitably increase over the coming years, designing a remote healthcare employee resource is a practical option where AI analysis can be conducted at a centralized facility. Therefore, an IoT-based solution coupled with BMM principles is key to managing motion capture resources. Hospitals and medical clinics can achieve positive employee productivity through training resources coupled with IoT solutions.

The IoT BMM platform must encapsulate the known possible postures when carrying heavy objects. The AIaaS and MLaaS backend infrastructure would contain the comprehensive training database capturing the main points where injury risk is known for an integrated medical facility. 
The main points that are captured from an IoT-based lifting rehabilitation framework can be summarized as follows [11]:

- The employee preparing to initially bend forward to the patient;

- The employee adjusting their gait to ensure a proper grip on the lying patient;

- The stage where load-bearing takes place, as the employee starts to lift the patient and perform the act of transference; and

- The final point where the employee places the patient on a different source, such as another bed, stretcher or wheelchair.

Risks of Repetitive Strain Injury (RSI) increase dramatically when improper lifting techniques are applied by healthcare employees. To counteract this problem, longer-term monitoring and training is essential to evaluate lifting practice regularly, not only to assess the capabilities of employees to carry properly but provide longitudinal health studies [4], [9].

The IoT platform must consider the use of client interfaces, such as mobile devices and tablets, to enable healthcare employees to interact with the training system. This provides a quantitative assessment of the lifting diagnosis of each employee, but remotely diagnose issues at the central training facility. Hence, the employee can proactively track their learning progress on their own device in conjunction with their local doctor.

Additionally, the healthcare employee would also allow for qualitative feedback to the IoT motion capture platform to ensure a virtuous loop for iterative improvement to the system. Consultation with user interactivity experts is important to design the platform in a manner that is beneficial to the employee and consultant physiotherapist who provide constructive feedback and ensure positive lifting practice.

The lifting technique framework should not be a means of punitive judgment, but rather an additional tool to offer continuous liaison with relevant healthcare stakeholders. The goal is to ensure harm minimization resulting from a back injury, while improving morale and workplace productivity through proper training methods.

\subsubsection{Combining IoT BMM with Human Activity Recognizers for Healthcare Employees}

The core IoT infrastructure deals with analyzing and recognizing human gait through specific motion capture algorithms. This offers results that are state-ofthe-art, especially for challenging gait recognition tasks processed in the AIaaS and MLaaS cloud-based computational layer:

- Classifying data sequences into key movements using the Histogram of Gradients (HoG) for human activity recognition; and 
- Adoption of neural network techniques to further optimize activity recognition by eliminating false positives.

The main aspect of the IoT BMM design is to incorporate lifting analysis for gait analysis by distinguishing on-field and off-field computational processing requirements methodically. These requirements can be distinguished as follows:

- Loading and preparing gait recognition information classified by time series, ensuring the data can be visually explored by the employee in consultation with their physiotherapist;

- Identified lifting issues are structured in a manner that allows employees to evaluate results while offering a guided reference for patient lifting best practices; and

- Offer potential for mobile deployment where network connectivity is intermittent or unreliable, thus improving the portability of the IoT platform in remote areas.

The issues of understanding the kinematic actions of the healthcare employee must be factored into the research, especially when multiple objects are involved. Visual interference impacts recognition accuracy, so this concern must be considered for additional testing purposes when the platform is rolled out for a healthcare workplace.

Furthermore, research must also consider support vector machine or neural network approaches when the employee undertakes a maneuver and adjusts their position for a specific context, such as distinguishing the difference between carrying a child or an elderly patient.

\subsubsection{Development Approach and Current Results}

The development of the lifting analysis platform involved a hybridized approach where cloud services were augmented with the local client infrastructure. This method sought to balance the needs of the end-user while satisfying the needs of the medical institution where the experiment was conducted. The biomimetic design principles used to design the platform fulfilled the following system requirements:

\section{Local Client Processing}

- Identify components that required immediate local processing, in order to serve immediate client needs:

- Data feeds that must be immediately presented to the end-user due to minimum latency needs, such as VR imaging presented to the medical patient; 
- Data that cannot be transmitted due to potential network bandwidth limitations or restrictions, especially where signal strength is weak for a wireless Internet connection.

\section{Remote Cloud Processing}

- Determine main features or functions that can be processed off-site onto the cloud infrastructure:

- Data points that can be safely backed up in order to prevent information loss due to unforeseen circumstances, such as the case where the laptop computer is accidentally lost whilst in transit between medical facilities;

- Data elements that can be processed remotely in order to mitigate processing constraints on the local computer since the laptop will have processing power limitations compared to a desktop workstation.

Table 3 summarizes the component-level identification of the rehabilitation platform regarding operational requirements:

Table 3

Hybrid Architecture for Rehabilitation Platform sourced from [13]

\begin{tabular}{|c|c|c|}
\hline Operational Location & Platform Components & Reasoning/Rationale \\
\hline $\begin{array}{l}\text { Local } \\
\text { Operations }\end{array}$ & $\begin{array}{l}\text { - Oculus Rift VR and } \\
\text { Leap motion sensor } \\
\text { hardware in C\#: } \\
\begin{array}{l}\text { Unity } \\
\text { 2018.1.6; }\end{array} \\
\text { MonoDevelop } \\
\quad \text { environment; } \\
\text { Blender graphics } \\
\text { toolkit. }\end{array}$ & $\begin{array}{l}\text { - Graphics processing } \\
\text { and rendering of the } \\
\text { gait simulation and } \\
\text { training was done on } \\
\text { the experimental kit; } \\
\text { - VR gear and laptop } \\
\text { were running directly } \\
\text { at the external medical } \\
\text { premises. }\end{array}$ \\
\hline $\begin{array}{l}\text { Remote } \\
\text { Operations }\end{array}$ & $\begin{array}{l}\text { - } \text { Remotely } \text { hosted } \\
\text { Cloud Infrastructure: } \\
\circ \quad \text { Fully managed } \\
\text { virtual machine } \\
\text { platform }\end{array}$ & $\begin{array}{l}\text { - Safely backup of } \\
\text { statistical data; } \\
\text { - Patient information } \\
\text { deleted prior to cloud } \\
\text { storage other than user } \\
\text { ID assignment. }\end{array}$ \\
\hline
\end{tabular}

As the rehabilitation exercises were conducted, the patients were assigned grades for their motor coordination ability, determined by their ability to conduct a routine grasping task of an object in space. Notably, while improvements were not exhibited by all patients, the overall trend indicates increasing familiarity with Virtual Reality technology by the patients enhances their user experience and positive engagement.

From the in-depth results examined in detail by Marzec et al. [13], a nominal improvement was observed especially for patients who had difficulty releasing objects in an active manner, as they were able to release objects after comprehensively using the VR rehabilitation platform as observed in Table 4. 
Thus, there is promise in the design principles of the experimental environment warranting further study for the combined approach of simulated and physical rehabilitation of poststroke patients.

Table 4

Poststroke Rehabilitation Observations extracted from [13]

\begin{tabular}{|l|l|l|}
\hline Patient & $\begin{array}{l}\text { Initial State Prior to VR } \\
\text { Training }\end{array}$ & Final State After VR Training \\
\hline Case I & $\begin{array}{l}\text { Actively releasing grasp/letting go } \\
\text { of object }\end{array}$ & $\begin{array}{l}\text { Actively releasing grasp/letting go } \\
\text { of object }\end{array}$ \\
\hline Case II & Not actively letting go of object & Actively releasing grasp of object \\
\hline Case III & Not actively letting go of object & Actively releasing grasp of object \\
\hline
\end{tabular}

The experimental limitations for future consideration include the need for data security and increasing the test sample size to verify the efficacy of the rehabilitation approach [13]. Data security is essential to ensure personally identifiable information is maintained for patient privacy, through Public-key Cryptography, so that the patient and doctor are only privy to such information. Furthermore, as the experiment is extended to more patients, the end results will verify the platform and its hybridized modular architectural approach.

\section{Conclusions}

This paper aims to provide an overview and analyze the benefits of adopting the biomimetic approach to middleware for IoT-based platforms, with an emphasis on dealing with the dynamic relationship between on-field and off-field machine learning functions. The data processing quality of the IoT sensor source, such as motion data captured from medical employees, should consider qualitative and quantitative user concerns to ensure broad acceptance of IoT in society while also mitigating apprehension to new technology.

End-user needs should always be accommodated when designing IoT biomimetic middleware as a quality attribute. This is achieved by knowing the domain context so that IoT middleware design composition manages heterogeneous IoT integration and accommodates end-user needs of security, reliability, and usability. Thus, this ensures the wide adoption of IoT-based BMM for a variety of different system frameworks.

The principles of biomimetics offer the primary framework of connecting different components within a context that is user-centric while accommodating future advances in IoT technologies that influence system design and architecture - this is examined by the case study with the emergence of consumer motion capture systems.

The study highlights the essential drivers for a common IoT biomimetic middleware framework, along with the design principles for future IoT applications. The flow of information from architectural through to biomimetic 
design show that IoT is the critical path as next-generation cellular networks guide tomorrow's information economy.

In conclusion, the understanding of complementary approaches, including learning-based ML methodologies such as neural networks and genetic algorithms, as well as training datasets, have a real-world impact on the operations of multi-modal IoT systems. Furthermore, performance optimization is needed for biomimetic design principles governing IoT infrastructure management.

\section{References}

[1] Bantz, D. F., Bisdikian, C., Challener, D., Karidis, J. P., Mastrianni, S., Mohindra, A., Shea, D. G., Vanove, M. (2003), Autonomic personal computing, Autonomic Computing, Vol. 42, No. 1, IBM Press

[2] Bass, L., Clements, P., Kazman, R. (2012), Software Architecture in Practice, $3^{\text {rd }}$ Edition, Boston: Addison-Wesley. ISBN 978-0-321-81573-6

[3] Britton, C., Bye P. (2004), IT Architectures and Middleware: Strategies for Building Large, Integrated Systems, Addison Wesley Professional

[4] Carneiro, P., Braga, A. C., Barroso, M. (2017), Work-related musculoskeletal disorders in home care nurses: Study of main risk factors, International Journal of Industrial Ergonomics, 61: 22-28, http://doi.org/10.1016/j.ergon.2017.05.002

[5] Chaczko, Z. C. (2009), Towards Epistemic Autonomy in Adaptive Biomimetic Middleware for Cooperative Sensornets. $\mathrm{PhD}$ Thesis, University of Technology, Sydney, Australia

[6] Chaczko, Z. C., Aslanzadeh, S. (2011), C2EN: Anisotropic Model of Cloud Computing, International Conference on Systems Engineering, Proceedings of $21^{\text {st }}$ International Conference on Systems Engineering, ICSEng 2011, Las Vegas, USA, pp. 467-473

[7] Chaczko, Z. C., Chiu, C., Aslanzadeh, S., Dune, T. (2011), Software Infrastructure for Wireless Sensor and Actuator Networks, Proceedings of $21^{\text {st }}$ International Conference on Systems Engineering, ICSEng 2011, Las Vegas, pp. 474-479

[8] Clements, P., Bachmann, F., Bass, L., Garlan, D., Ivers, J., Little, R., Merson, P., Nord, R., Stafford, J. (2010), Documenting Software Architectures: Views and Beyond, $2^{\text {nd }}$ Edition, Boston: Addison-Wesley. ISBN 978-0-321-55268-6

[9] European Council Directive - 90/269/EEC, European Union Health and Safety Requirements for Manual Load Handling, http://eurlex.europa.eu/legal-content/EN/TXT/?uri=CELEX\%3A31990L0269

[10] Godinez, M., Hechler, E., Koenig, K., Lockwood, S., Oberhofer, M., Schroeck, M. (2010), The Art of Enterprise Information Architecture: A Systems-Based Approach for Unlocking Business Insight, IBM Press 
[11] Health and Safety Executive, U.K. Government, Moving and Handling Case Studies in Health and Social Care, http://hse.gov.uk/healthservices/moving-handling-case-studiesresearch.htm

[12] Krakowiak, S. (2009), Middleware Architecture with Patterns and Frameworks

[13] Marzec, M., Olech, M., Klempous, R., Nikodem, J., Kluwak, K., Chiu, C., Kołcz, A. (2019), Virtual Reality Poststroke Rehabilitation with Localization Algorithm Enhancement, Virtual and Augmented Reality in Education Conference, Lisbon, Portugal, http://www.mscles.org/conf/vare2019/

[14] McConnell, S. (1999), After the Goldrush - Creating a True Profession of Software Engineering, Microsoft Press

[15] Pressman, R., Maxim, B. (2014), Software Engineering: A Practitioner's Approach, $8^{\text {th }}$ Edition, McGraw Hill

[16] Systems Engineering Competency Maturity Model - Architecture Papers (1995), Software Engineering Institute, Carnegie Mellon University

[17] McGill, S. (2015) Back mechanic: the secrets to a healthy spine your doctor isn't telling you: the step-by-step McGill method to fix back pain, p. 2 